[2] N: A. Fava, Weals type inequalities for product operators, ibid. 42 (1972), 271-28s.

[3] M. de Guzmán, Differentiation of Integrals in $R^{n}$, Springor-Vorlag, Vol. 481.

[4] E. Stein, Note on the class $L\left(\log ^{+} L\right)$, Studia Math. 32 (1969), 305-310.

Received Mareh 16, 1982

Revised version June 1, 1982

(1744)

\section{On a theorem of Lebow and Mlak for several commuting operators}

by

J. JA N A S (Kraków)

Abstract. A result of Mlak coneerning the spectral radius of an operator in a Hilbert space is extended to several commuting operators.

Let $H$ be a complex Hilbert space. Denote by $L(H)$ the Banach algebra of all bounded linear operators in $H$. For an $n$-tuple of pairwise commuting operators $T_{1}, \ldots, T_{n}$ with the Taylor joint spectrum $\sigma\left(T_{1}, \ldots\right.$ $\left.\ldots, T_{n}\right)$ contained in the open unit ball $B \subset C^{n}\left(B=\left\{z \in C^{n},|z|<1\right\}\right)$ we denote by

$$
M(\xi, T)=I-\sum_{i=1}^{n} \xi_{i} T_{i}, \quad \xi \in \partial B
$$

the topological boundary of $B$.

Note that $M(\xi, T)$ is invertible for every $\xi \in \partial B$ (by the spectral mapping theorem for $\left.\sigma\left(T_{1}, \ldots, T_{n}\right)\right)$.

The operator-valued function $M(\xi, T)^{-n}$ plays the role of the Fredholm resolvent for the above system $T_{1}, \ldots, T_{n}$. In fact, it is easy to prove that for every function $f$ holomorphic in $B$ and continuous in $\bar{B}$ (the closure) we have the equality

$$
f\left(T_{1}, \ldots, T_{n}\right)=\int_{\partial B} M(\xi, T)^{-n} f(\xi) \Omega(\xi),
$$

where $\Omega(\xi)$ is the $(n-1, n)$ differential form given explicitly by Henkin; see [6] for the definition.

Let us recall some definitions and notations. Denote by $U$ $=\{z \in C,|z|<1\}$ the open unit disc. For $p \geqslant 1$ and $\alpha \geqslant 0$ let

$$
A^{p, a}=\left\{f, f: U \rightarrow C \text { is holomorphic and } \int_{U}|f|^{p}\left(1-|z|^{2}\right)^{a} d x d y<+\infty\right\} .
$$

For $f \in A^{p, \alpha}$ let $\|f\|_{p, \alpha}^{p}=\int_{U}|f|^{p}\left(1-|z|^{2}\right)^{\alpha} d x d y$. The space $A^{p, a}$ is called the Bergman space and has been investigated in detail by Horowitz [2], [3] 

space

Let $\sigma$ be the surface Lebesgue measure on $\partial B$. We define the Hardy

$H^{p}(B)=\left\{f, f: B \rightarrow C\right.$ is holomorphic and $\left.\sup _{0<r<1} \int_{\partial B}|f(r z)|^{p} d \sigma(z)<\infty\right\}$.

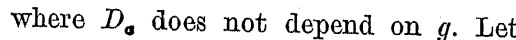

$$
g_{1}(z)=\sum_{k} b_{k_{i}} z^{k}, \quad g_{2}(z)=\sum_{k} c_{k} z^{k^{c}}
$$

By using the polar coordinates it is easy to check the following

Proposimion 1. We have $g \in H^{p}(B)$ iff for every $\lambda \in \partial B$ the function $g_{\lambda}(w)=g\left(\lambda_{1} w, \ldots, \lambda_{n} w\right), w \in U$, belongs to $A^{p, n-2}, n \geqslant 2$.

Now let us recall the Hardy inequality. Let $h \in H H^{1}(U)$ and $h(z)=$ $=\sum_{k \rightarrow 0} c_{k} z^{r_{6}}$; then $\sum_{k}\left|c_{k}\right| k^{-1}<+\infty$, see [1]. Now we shall give an analoguous inequality for $g \in A^{1, n-2}$.

Proposirion 2. If $g \in A^{1, n-2}(n \geqslant 2)$ and $g(z)=\sum_{k} a_{k c^{2}} z^{h}$, then $\sum_{k}\left|a_{k i}\right| k^{-n}$ $<+\infty$.

Proof. Denote $n-2=\alpha$. Following the proof of the Hardy inequality given in [1] we first assume that $a_{k} \geqslant 0, \forall k$. Now

$$
\operatorname{Im} g\left(r e^{i \theta}\right)=\sum_{m=1}^{\infty} a_{m} r^{m} \sin m \theta, \quad 0 \leqslant r<1 .
$$

Hence

$$
\sum_{m=1}^{\infty} m^{-1} a_{m} r^{m} \leqslant(1 / 2) \int_{0}^{2 \pi}\left|g\left(r e^{i \theta}\right)\right| d \theta .
$$

Multiply both sides of the last inequality by $\left(1-r^{2}\right)^{\alpha_{1}}$ and integrate over $(0,1)$. Then we have

$$
\sum_{m=1}^{\infty} m^{-1} a_{m} \int_{0}^{1} r^{m}\left(1-r^{2}\right)^{\alpha} r d r \leqslant(1 / 2)\|g\|_{1, \alpha} .
$$

But

$$
\int_{0}^{1} r^{m}\left(1-r^{2}\right)^{a} r d r \geqslant C_{n} m^{1-n} \quad \text { for a certain } C_{n} \text { and } m=1,2, \ldots
$$

Thus

$$
C_{n} \sum_{m=1}^{\infty} a_{m} m^{-n} \leqslant(1 / 2)\|g\|_{1, \alpha} .
$$

If $g \in A^{1, a}$ is arbitrary, then by Theorem 1 of [3] there exist $g_{1}, g_{2} \in A^{2, a}$ such that $g=g_{1} g_{2}$ and.

$$
\left\|g_{1}\right\|_{2, a}\left\|g_{2}\right\|_{2, \alpha} \leqslant D_{a}\|g\|_{1, \alpha},
$$

$$
G_{1}=\sum_{k}\left|b_{k}\right| z^{k}, \quad G_{2}=\sum_{k}\left|c_{k}\right| z^{k}
$$

also belong to $A^{2, \alpha}$ and $\left\|g_{s}\right\|_{2, \alpha}=\left\|G_{s}\right\|_{2, \alpha}, s=1,2$. Put $H=G_{1} G_{2}$. Then $H \in A^{1, a}$. But

$$
H=\sum_{k}^{i} \tilde{a}_{k} z^{k}, \quad \tilde{a}_{k} \geqslant 0 \quad \text { and } \quad\left|a_{k}\right| \leqslant \tilde{a}_{k}, \quad \forall k .
$$

Applying (*) to $H$ we have

$$
\sum_{k}\left|a_{k}\right| k^{-n} \leqslant \sum_{k} \tilde{a}_{\pi_{k}} k^{-n} \leqslant O_{n}\|H\|_{1, \alpha} .
$$

Since

$$
\|H\|_{1, \alpha} \leqslant\left\|G_{1}\right\|_{2, \alpha}\left\|G_{2}\right\|_{2, \alpha}=\left\|g_{1}\right\|_{2, \alpha}\left\|g_{2}\right\|_{2, \alpha} \leqslant D_{a}\|g\|_{1, \alpha},
$$

and so the proof is complete.

Before we proceed further let us recall the above-mentioned result. of Mlak [5]. Let $T$ be an operator in a complex Hilbert space $H$. Assume that $\left((I-z T)^{-1} x, y\right), z \in U$, belongs to $H^{1}(U)$ for every $x, y \in H$. Then $r(T)<1$, where $r(T)$ is the spectral radius of $T$. Mlak's result extends an earlier theorem of Lebow [4]. Here is a generalization of Mlak's theorem to higher dimensions. Let $T_{1}, \ldots, T_{n}$ be a system of commuting operators in $H$. First of all note that the following extension of the above result. is obvious.

$$
\text { If }\left(\prod_{i=1}^{n}\left(I-z_{i} T_{i}\right)^{-1} x, y\right) \in H^{1}\left(U^{n}\right), \text { then } r\left(T_{i}\right)<1, i=1, \ldots, n \text {. }
$$

This is immediate by Mlak's theorem. But we also have the following generalization:

Proposimion 3. Let $T_{1}, \ldots, T_{n}$ be a commuting system of operators in a complen Hitbert space $H, n \geqslant 2$. Assume that $\left(M_{1}(\bar{z} ; T)^{-n} x, y\right) \in H^{1}(B)$ for overy $x, y \in H$. Then

$$
\sigma\left(T_{1}, \ldots, T_{n}\right) \subset \mathcal{B},
$$

where $\sigma\left(T_{1}, \ldots, T_{n}\right)$ denotes the Taylor joint spectrum of $T_{1}, \ldots, T_{n}$. Proof. By Proposition 1 we have

$$
\left(M(\bar{z}, T)^{-n} x, y\right) \in H^{1}(B) \Leftrightarrow \forall \xi \in \partial B,\left(M(\xi, w T)^{-n} x, y\right) \in A^{1, n-2} .
$$


But it is clear that

$$
\sigma\left(T_{1}, \ldots, T_{n}\right) \subset B \Leftrightarrow \forall \xi \in \partial B, r\left(\sum_{i} \xi_{i} T_{i}\right)<1 .
$$

Fix $\dot{\xi} \in \sigma B$ and let $T_{\xi}=\sum_{i} \xi_{i} T_{i}$. We know that $\left(\left(I-w T_{\xi}\right)^{-n} x, y\right) \in A^{\mathrm{i}, n-2}$. Suppose that there exists a $\lambda \in \partial U$ and $\bar{\lambda} \in \sigma\left(T_{\xi}\right)$. Put $S_{\xi}=\bar{\lambda} T_{\xi}$. We have $1 \in \sigma\left(S_{\xi}\right)$ and $\left(\left(I-w S_{\xi}\right)^{-n} x, y\right) \in A^{1, n-2}$. Since

$$
\left(\left(I-w S_{\xi}\right)^{-n} x, y\right)=\left(\sum_{k=1}^{\infty}\left(\begin{array}{c}
n+7 w-1 \\
n-1
\end{array}\right)\left(w S_{\xi}^{S_{\xi}}\right)^{k} x, y\right)
$$

applying Proposition 2 we can write

$$
\begin{aligned}
\left|\left(\sum_{k=1}^{s} k^{-n}\left(\begin{array}{c}
n+7 c-1 \\
n-1
\end{array}\right) S_{\xi}^{k} x, y\right)\right| & \leqslant \sum_{k=1}^{\infty}\left|\left(k^{-n}\left(\begin{array}{c}
n+70-1 \\
n-1
\end{array}\right) S_{\xi}^{l o} x, y\right)\right| \\
& \leqslant I r_{x, y}<+\infty .
\end{aligned}
$$

Hence the sequence of operators

$$
R_{s}=\sum_{l_{i}=1}^{s} k^{-n}\left(\begin{array}{c}
n+k-1 \\
n-1
\end{array}\right) S_{s}^{k}
$$

is bounded in norm, i.e., $\left\|R_{s}\right\|<M<+\infty, s=1,2, \ldots$

Denote by $\mathscr{P}$ the Banach algebra generated by $S_{\xi}$ and $I$. Then thore exists a $\eta \in \mathbb{S p} \mathscr{P}$ such that $\eta\left(S_{\xi}\right)=1$. Hence $\left|\eta\left(R_{s}\right)\right| \leqslant\left\|R_{s}\right\|<\mathbb{M}, s$ $=1,2, \ldots$

But on the other hand

$$
\begin{gathered}
\eta\left(R_{s}\right)=\sum_{k=1}^{s} k^{-n}\left(\begin{array}{c}
n+k-1 \\
n-1
\end{array}\right) \eta\left(S_{\xi}\right)^{k}=\sum_{k=1}^{s} k^{-n}\left(\begin{array}{c}
n+k-1 \\
n-1
\end{array}\right) \\
>\sum_{k=1}^{s} k^{-n} k^{n-1}=\sum_{k=1}^{s} k^{-1}, \quad s=1,2, \ldots
\end{gathered}
$$

'This contradiction completes the proof.

Remark 1. It is clear that the same result also holds for an $n$-tuple of commuting operators in a complex Banach space.

Remark 2. Proposition 3 can be extended to more general domains in $\boldsymbol{C}^{n}$, as a result of certain integral formulas, but this will be shown in another paper.

Note added in proof. Applying a method of Nikolski we have extended the abovo result to a more general context (to appear in Bull. Acad. Polon. Sci.).

\section{References}

[1] K. II offmin, Banach spases of analytic functions, Now York 1962.

[2] C. Horowit\%, Zeros of functions in the Bergman spaces, Duks Math. J. 41 (1974), (3):3-710.

[3] - Fuetorization theorems for funclions in the Bergman spaces, ibid. 44 (1977), $201-213$.

[4] A. Tiobow, Spestral radius of an absolutoly continuous operator, Proc. Amor. Mitith. Soc. 206 (1973).

[5] W. Mlak K, On a liteorem of Lebow, Ann. Polon. Math. 35, 1. (1977), 107-109.

[(i] 11. W. Suabut, Introtuction to complex analysis, Warsaw 1.974 (in Polish).

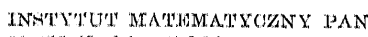

31-027 kinków, Solsklugo 30

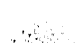

